\title{
Clinical efficacy of OASIS Preloaded Punctal Plug in Comparison to Smart Plug Punctal Plug in Aqueous- Deficient Dry Eye Disease
}

Fei Luo

Beijing Tongren Hospital

Lei Tian

Beijing Tongren Hospital

Shijing Deng

Beijing Tongren Hospital

Jie Hao

Beijing Tongren Hospital

ying jie ( $\nabla$ jieying202010@126.com )

Beijing Tongren Hospital

\section{Research}

Keywords: Preloaded punctal plug, dry eye, Smart Plug punctal plug, embolization implantation, OSDI questionnaire

Posted Date: June 23rd, 2021

DOI: https://doi.org/10.21203/rs.3.rs-626016/v1

License: (c) (i) This work is licensed under a Creative Commons Attribution 4.0 International License. Read Full License 


\section{Abstract}

\section{Background}

At present, there are few clinical studies comparing OASIS preloaded punctal plug and Smart Plug punctal plug for dry eye. This study intends to evaluate the effect of OASIS preloaded punctal plug versus Smart Plug punctal plug in the treatment of aqueous-deficient Dry Eye.

\section{Methods}

47 patients with aqueous-deficient dry eye were selected and randomly divided into control group and experimental group. The control group was treated with Smart Plug punctal plug treatment, and the experimental group was treated with OASIS preloaded punctal plug treatment. The OSDI questionnaire score, Schirmer I test and BUT results before and after treatment, and the incidences of postoperative complications were compared between the two groups. Follow up for six months.

\section{Results}

The results of this study showed that compared with before treatment, the OSDI scores of patients were significantly improved at 6 months after treatment in both the experimental group and control group. After treatment, there was no significant difference in OSDI score, Schirmer I test, and BUT level between the two groups. Besides, the Smart Plug punctal plug treatment group had a significant improvement in BUT at 3 months after operation compared with before treatment.

\section{Conclusion}

The OASIS preloaded punctal plug is comparable to the Smart Plug punctal plug in treating aqueousdeficient dry eye, and both can significantly improve dry eye symptoms. Furthermore, the OASIS preloaded punctal plug can facilitate intraoperative procedures, lacrimal punctum expansion and plug implantation can be completed in one step, and the loss of embolization before implantation and the abnormal implantation due to the expansion of the embolic volume can be reduced, which is worthy of clinical application.

Clinical registration number: researchregistry6629; Registered 4 March 2021 - Retrospectively registered

\section{Background}

Dry eye is a chronic ocular surface disease caused by multiple factors. It is caused by the instability of the tear film or the imbalance of the ocular surface microenvironment caused by the abnormal quality, quantity and dynamics of the tear fluid. It can be accompanied by ocular surface inflammation, tissue damage and neurological abnormalities, causing various ocular discomforts and/or visual dysfunction [ ${ }^{1}$,

2]. The incidence of dry eye in China is similar to that of other Asian countries and is higher than that of the United States and Europe, and its incidence is about $21 \% \sim 52.4 \%{ }^{[3,4]}$. According to the components 
of the tear film, dry eyes are divided into the aqueous-deficient dry eye, abnormal lipid dry eye, abnormal mucin dry eye, abnormal tear motility dry eye, and mixed dry eye. Among them, the Aqueous-Deficient Dry Eye can be divided into Sjögren dry eye and without Sjögren dry eye ${ }^{[5,6]}$.

Currently, the therapeutic options for dry eye include artificial tears supplement, lacrimal duct plug implantation, and submandibular gland transplantation ${ }^{[7,8]}$. In recent years, Smart Plug punctal plug has become more frequently used to treat aqueous-deficient dry eye, by blocking the tear dots of patients to prevent the discharge of tears, the normal secretion of tears is retained in the eyes, thereby improving symptoms and achieving good curative effect ${ }^{[9,10,11]}$. However, the complications of lacrimal duct plug, especially those of long-term follow-up, have not yet attracted enough attention ${ }^{[12,13]}$. OASIS preloaded punctal plug as a new type of lacrimal duct plug that has attracted significant interest,however, there are few clinical studies comparing OASIS preloaded punctal plug and Smart Plug punctal plug for dry eye. Therefore, in this study, patients with Aqueous-Deficient Dry Eye who were treated in our hospital from January 2018 to January 2020 are the main research objects, and we intend to explore the clinical efficacy of Oasis preloaded punctal plug in the treatment of Aqueous-Deficient Dry Eye.

\section{Materials And Methods}

\section{Research objects}

A randomized, controlled, prospective study was performed. 47 patients with the aqueous-deficient dry eye at our hospital from January 2018 to January 2020 were taken as the main research objects. The included patients were randomly divided into control group and experimental group in the form of randomized cards (24 in the experimental group, 23 in the control group). Patients in the control group were treated with Smart Plug punctal plug, and patients in the experimental group were treated with OASIS preloaded punctal plug. This study complies with the "Declaration of Helsinki of the World Medical Association" and has been approved by the ethics committee of our hospital. All patients signed an informed consent form.

\section{Inclusion and exclusion criteria}

Inclusion criteria: (1) Patients who meet the following criteria and with severe dry eyes in both eyes were included in the study. A: One of the subjective symptoms such as dryness, foreign body sensation,

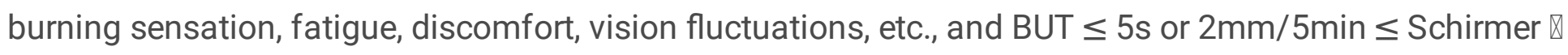
test (no topical anesthesia) $\leq 5 \mathrm{~mm} / 5 \mathrm{~min}$; B: one of the subjective symptoms such as dryness, foreign body sensation, burning sensation, fatigue, discomfort, vision fluctuation, and $5 \square B U T \leq 10$ or $5 \mathrm{~mm} / 5 \mathrm{~min} \square$ Schirmer $\rrbracket$ test (without topical anesthesia) $\leq 10 / 5 \mathrm{~min}$, at the same time corneal fluorescein staining is positive; (2) Patient who are between 10 and 70 years old; (3) The lacrimal duct is unobstructed; (4) Patients who have signed informed consent.

Exclusion criteria: (1) Patients with lacrimal duct disease; (2) Patients with abnormal ocular surface structure; (3) Patients with active ocular surface inflammation. 


\section{Research methods}

The experimental group used the 6303 preloaded punctal plug produced by OASIS Medical (Fig. 1), and the control group used the Smart Plug punctal plug. Before the operation, the two groups of patients flushed the lacrimal duct, applied Alkain surface anesthesia, under the microscope, lightly pressed the eyelid to expose the punctum (if the punctum is too small, the punctum can be expanded), and placed the brown tip of the implanter at the punctum and squeezed the implanter so that the preloaded punctal plug entered the lacrimal canaliculus from the tip of the implanter. The top edge of the plug should be below the punctum. If necessary, push the punctal plug further with micro tweezers to reach the level of the canaliculus. One drop of levofloxacin eye drops was applied after the operation. Ofloxacin eye drops were applied to the eyes for 3 days. Smart Plug punctal plug in operation method: wash lacrimal duct without the obstruction of lacrimal duct, proparacaine hydrochloride eye drops were applied for ocular surface anesthesia, the lower eyelid was pulled apart to exposed lacrimal punctum, According to the size of the lower lacrimal punctum, expand it with the punctum dilator, the other hand held embedded tweezers along the tooth groove clamp Smart Plug punctal plug, the other end is quickly inserted vertically along the lacrimal duct about $2 / 3$ of the length of the plug. Release the embedded tweezers and observe the remaining plug retracting completely into the lacrimal site, Ofloxacin eye drops were applied to the eyes for 3 days. Follow up for six months.

\section{Main observation indicators and methods}

Before the lacrimal canalicular embolization and 1 week, 1 month, 3 months, and 6 months after the embolization, the treated patients were successively surveyed with OSDI scores. Eye examinations include: visual acuity examination, intraocular pressure, slit lamp examination, and corneal fluorescein staining, lissamine green staining of conjunctiva, BUT, Schirmer I test (without surface anesthesia). The corneal fluorescein staining score was scored using a 0-12 point system to record the staining results (the cornea was divided into 4 quadrants, and each quadrant was divided into 0 to 3 points according to the degree of staining and the staining area). All tested patients were completed by the same doctor in the same examination room, and the result was the average of 3 repeated examinations.

\section{Statistical methods}

In this study, SAS 9.4 statistical software was used for data processing, and the measurement data were

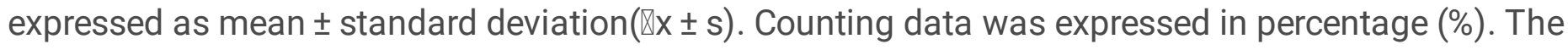
comparison between the experimental group and the control group of each index used a mixed-effect linear model, and $p<0.05$ was considered to be statistically significant; the experimental group and the control group of each index were compared with a mixed-effect linear model at different time points. A Chi-square test was used for counting data. As it involves many comparisons and correction of test level, $P<0.005$ is considered to be statistically significant.

\section{Results}




\section{General information}

A total of 47 patients with water-deficiency dry eye were included in this study, including 6 males (6 eyes) and 41 females (41 eyes), aged 26 to 70 years, with an average age of 56.06 years, and the duration of the disease was 6 months to 36 months. The clinical follow-up time is 6 months.

\section{Comparison of OSDI scores between the two groups}

In the OASIS preloaded punctal plug treatment group, OSDIO and OSDI2, OSDI3 and OSDI4 were different; OSDI1 and OSDI2, OSDI3 and OSDI4 were different, and OSDI4 and OSDI2 were different, and there was no statistical difference between the other time points. In the Smart Plug punctal plug treatment group, OSDIO and OSDI1, OSDI2, OSDI3, and OSDI4 were different; OSDI1 and OSDI2, OSDI3, and OSDI4 were different, and OSDI2 was different from OSDI3 and OSDI4, and there was no difference between other time points. Statistical differences. Compared with before treatment, the OSDI score of patients in the OASIS preloaded punctal plug treatment group was significantly improved 6 months after treatment, and the difference was statistically significant $(p<0.05)$. There was no significant difference in OSDI scores between the experimental group and the control group 6 months after treatment $(p=0.53)$. See Fig. 2 .

\section{Comparison of the results of the Schirmer $₫$ test between the two groups}

In the OASIS preloaded punctal plug treatment group, the results of the Schirmer $\nabla$ test were not statistically different at each time point. In the Smart Plug punctal plug treatment group, there was a statistical difference between SCHIMMER1 and SCHMMER4, and there was no statistical difference between the other time points. There was no statistical difference between the experimental group and the control group in the Schirmer $\otimes$ test 6 months after treatment $(p=0.41)$. See Fig. 3.

\section{Comparison of BUT results between the two groups}

There was no statistically significant difference in BUT levels among patients in the OASIS preloaded punctal plug treatment group. In the Smart Plug treatment group, there was a statistical difference between BUTO and BUT3, and there was no statistical difference at other time points. There was no significant difference in BUT levels between the experimental group and the control group 6 months after treatment $(p=0.35)$. See Fig. 4 .

\section{Comparison of postoperative complications between the two groups}

One patient in the OASIS preloaded punctal plug treatment group developed conjunctivitis on the second day after implantation and recovered after 4 days of drug treatment. There were no abnormal complications in the Smart Plug punctal plug treatment group. 


\section{The intraoperative and postoperative complications}

The intraoperative and postoperative complications are shown in Table 1, all symptoms were relieved after symptomatic treatment.

Table 1

The intraoperative and postoperative complications of two groups.

\begin{tabular}{|c|c|c|}
\hline group & intraoperative & postoperative \\
\hline \multirow[t]{3}{*}{$\begin{array}{l}\text { The control } \\
\text { group }(n= \\
\text { 23) }\end{array}$} & $\begin{array}{l}\text { (1) Overexpansion occurred in } 2 \text { cases } \\
\text { with lower lacrimal punctum laceration }\end{array}$ & $\begin{array}{l}\text { (1) } 2 \text { cases had redness and swelling of } \\
\text { lower lacrimal punctum one day after } \\
\text { operation }\end{array}$ \\
\hline & $\begin{array}{l}\text { (2) In } 2 \text { cases, the plug was not } \\
\text { implanted for the first time due to the } \\
\text { rapid expansion of punctal plug, and the } \\
\text { new plug was implanted again }\end{array}$ & $\begin{array}{l}\text { (2) } 1 \text { case had epiphora one week after } \\
\text { operation (withdrawal from clinical) }\end{array}$ \\
\hline & $\begin{array}{l}\text { (3) In } 1 \text { case, the plug was lost because } \\
\text { the embedded tweezers were not aligned } \\
\text { with the end of the plug, and a new plug } \\
\text { was re-implanted }\end{array}$ & \\
\hline \multirow{2}{*}{$\begin{array}{l}\text { The } \\
\text { experimental } \\
\text { group }(n= \\
24)\end{array}$} & - & $\begin{array}{l}\text { (1) } 2 \text { cases had redness and swelling of } \\
\text { lower lacrimal punctum one day after } \\
\text { operation }\end{array}$ \\
\hline & & $\begin{array}{l}\text { (2) } 1 \text { patient presented lower lacrimal } \\
\text { punctum redness and swelling } \\
\text { accompanied by conjunctival } \\
\text { congestion (withdrawal from clinical) }\end{array}$ \\
\hline
\end{tabular}

\section{Discussion}

A total of 47 patients with aqueous-deficient dry eye were included in this study. The results of the study showed that compared with before treatment, the OSDI scores of patients in the OASIS preloaded punctal plug treatment group and Smart Plug punctal plug treatment group were significantly improved 6 months after treatment. After treatment, the two groups of patients were similar in OSDI score, Schirmer I test, and BUT levels, and there was no significant difference.

Dry eye is a common and frequently occurring disease in ophthalmology ${ }^{[14]}$. Common symptoms include dry eyes, easy tiredness, itchy eyes, foreign body sensation, burning sensation, sticky discharge, fear of wind, photophobia, and sensitivity to external stimuli. Sometimes the eyes are too dry and there is not enough tear fluid, but it stimulates the secretion of reflex tears, causing frequent tears. In more serious cases, the eyes will be red and swollen, congested, keratinized, corneal epithelial defects, and filaments sticking. This kind of damage can cause corneal and conjunctival lesions over time and affect vision [1517]. The International Dry Eye Workshop (DEWS) divides the disease into four grades according to its 
severity and treatment methods, and according to the different grades, it recommends the application of topical artificial tears, cyclosporine, tetracyclines, and lacrimal embolization ${ }^{[18]}$.

In recent years, with the aging of the population, the use of electronic devices, and environmental factors, the number of patients with dry eye has been on the rise ${ }^{[19-22]}$. At present, there are various ways to treat dry eye, mainly including the elimination of incentives, artificial tear replacement therapy, preservation of own tears, promotion of tear secretion, further combining anti-inflammatory and immunosuppression, and surgical treatments ${ }^{[23-25]}$. However, general incentives are difficult to eliminate, and due to the complex composition of human tears, although there are various artificial tears for different tear film components, they still cannot completely replace their own tears. The side effects of immunosuppressive agents are large and cost much ${ }^{[26]}$. Moreover, long-term topical medication, the preservatives in the medication can easily cause damage to the ocular surface. If the dry eye patients need to use artificial tears frequently, such as more than 4-6 times a day; if the application of artificial tears has side effects or patients cannot tolerate or do not accept long-term drug treatment; if the drug treatment effect is poor or the symptoms cannot be completely solved by using artificial tears alone, lacrimal embolization can be adopted.

The first lacrimal plug used is a degradable punctum plug for the treatment of the severe dry eye. Nowadays, the lacrimal plug has developed a variety of shapes and materials, and its application is becoming more and more extensive. Lacrimal embolism can be divided into punctal plug and canaliculus embolism according to its location, and the time of their placement is divided into temporary and permanent embolism. Embolism is made of different materials, and current reports include collagen, silica gel, hydrogel, polydioxanone, and acrylic acid, etc. ${ }^{[27]}$. Generally speaking, when local lubrication cannot be improved, these small plugs improve the signs and symptoms of moderate dry eye. Permanent plugs have a longer residence time, so their impact is usually greater than that of temporary degradable plugs. Punctal plug are well tolerated, only about $10 \%$ need to be removed due to irritation. The literature shows that compared with the lacrimal canalicular plug, the lacrimal punctum plug has a higher probability of lacrimal overflow and plug body loss. Permanent punctal plug are more prone to side effects such as lacrimal canaliculitis and pyogenic granuloma. For a small number of patients, more invasive treatments are needed to remove them, such as lacrimal canalitomy and dacryocystorhinostomy.

Smart Plug punctal plug has been used for many years and is effective in the treatment of aqueousdeficient dry eye ${ }^{[10,28]}$. The results of this study support the fact that Smart Plug punctal plug significantly improved subjective symptoms in patients with dry eye and prolonged BUT after surgery. However, Schirmer did not show significant improvement, presumably because punctal plug does not increase the secretion of the main and accessory lacrimal glands, thus increasing tear production. Instead, the symptoms of dry eye can be improved by reducing the limited tear drainage and increasing tear film stability. OASIS preloaded punctal plug is a new type of lacrimal duct plug, but high-quality clinical research articles are few currently. Our current study showed that that the OASIS preloaded 
punctal plug is comparable to the Smart Plug punctal plug in treating aqueous-deficient dry eye, and both can significantly improve dry eye symptoms. The OASIS preloaded punctal plug uses the device's own punctum dilator is a better match for the implant and reduces the risk of intraoperative overdilation of the lacrimal duct. Furthermore, it simplifies the surgical procedure, lacrimal punctum expansion and plug implantation can be completed in one step, the loss of embolization before implantation and the abnormal implantation due to the expansion of the embolic volume can be reduced, and minimize the risk of intraoperative complications. Therefore, it is worthy of clinical promotion.

Although the two kinds of lacrimal duct plugs had different degrees of postoperative complications, they were relieved by simple treatment, no serious irreversible complications occurred. Perhaps due to the short observation time, no canaliculus granuloma and canaliculitis requiring surgical treatment were observed. Whether there are serious complications in the later stage still needs Further observations.

This research still has the following shortcomings. First of all, although this study is a randomized controlled experiment, it is not blinded. Secondly, this study is a single-center clinical study, and the sample size included is relatively small. It is still necessary to increase the sample size and conduct multicenter clinical research. Finally, the clinical follow-up time of this study is relatively short, and long-term clinical follow-up observation is still needed.

\section{Conclusion}

The OASIS preloaded punctal plug can achieve similar therapeutic effects as the Smart Plug punctal plug, and because of the simple operation procedure of the preloaded punctal plug, it can complete the dilation and embolization of the lacrimal punctum in one step, and can reduce the loss of the embolization before the implantation and the abnormal implantation due to the expansion of the embolization volume, which is worthy of clinical application.

\section{Declarations}

\section{Ethics approval and consent to participate}

This study was conducted in accordance with the Declaration of Helsinki and approved by the ethics committee of Beijing Tongren Hospital (TREC2016-45). All patients signed an informed consent form. Written informed consent was obtained from a parent or guardian for participants under 16 years old.

\section{Consent for publication}

All authors agree to publish this article.

\section{Availability of data and materials}


The datasets used and/or analysed during the current study are available from the corresponding author on reasonable request.

\section{Competing interests}

All of the authors had no any personal, financial, commercial, or academic conflicts of interest separately.

\section{Funding}

This study was supported by National Natural Science Foundation of China (81970764). The National Natural Science Foundation of China had no role in the design, data collection, analysis, interpretation of data, and in writing the manuscript for publication.

\section{Authors' contributions}

FL, LT conceived of the study, and SJ and JH participated in its design and coordination and $\mathrm{YJ}$ helped to draft the manuscript. All authors read and approved the final manuscript.

\section{Acknowledgements}

Not applicable

\section{References}

1. Sheppard JD. Guidelines for the treatment of chronic dry eye disease. Manag Care. 2003;12:20 - 5 .

2. Craig JP. Nichols K K, Akpek EK, et al TFOS DEWS II Definition and Classification Report. Ocul Surf. 2017; 15(3): 276-83.

3. Jiang JX. Lin L M, Lian GL, et al Vitamin A deficiency and child feeding in Beijing and Guizhou, China. World Journal of Pediatrics. 2008; 4(1): 20-5.

4. Peng QH. Liu H Y, Yao XL, et al Effect of acupuncture on the regulation of sex hormone in patients with xerophthalmia. International Journal of Ophthalmology. 2009; 9(8): 1534-6.

5. Skalicky SE. Petsoglou C, Gurbaxani A, et al New Agents for Treating Dry Eye Syndrome. Current Allergy Asthma Reports. 2013; 13(3): 322-8.

6. Na KS. Mok J W, Kim JY, et al Proinflammatory gene polymorphisms are potentially associated with Korean non-Sjogren dry eye patients. Mol Vis. 2011; 17(306): 2818-23.

7. Marshall LL. Roach JM. Treatment of Dry Eye Disease. Consult Pharm 2016 Feb,31(2):96-106.

8. Simmons PA. Vehige JG. Clinical performance of a mid-viscosity artificial tear for dry eye treatment. Cornea. 2007;26(3):294-302.

9. Sakamoto A. Kitagawa K, Tatami A. Efficacy and retention rate of two types of silicone punctal plug in patients with and without Sjögren syndrome. Cornea. 2004;23(3):249 - 54. 
10. Balaram M. Schaumberg DA, Dana MR. Efficacy and tolerability outcomes after punctal occlusion with silicone plugs in dry eye syndrome. Am J Ophthalmol. 2001;131(1):30 - 6.

11. Gordonson LC. Efficacy and tolerability outcomes after punctal occlusion with silicone plugs in dry eye syndrome. Am J Ophthalmol. 2001;132(4):600-1.

12. Klein-Theyer A. Boldin I, Rabensteiner DF, et al Prevalence of canaliculitis after smartplug insertion during long-term follow-up. Br J Ophthalmol. 2015;99(8):1134-6.

13. Musadiq M. Mukherji S, Sandramouli S. Pyogenic granuloma following silicone punctal plug: report of two cases. Orbit. 2005;24(2):149 - 51.

14. Lemp MA. The 1998 Castroviejo Lecture. New strategies in the treatment of dry-eye states. Cornea. 1999;18(6):625 - 32.

15. Liu ZG. [Paying attention to the expert consensus on dry eye to standardize and promote the clinical diagnosis and treatment of dry eye]. Zhonghua Yan Ke Za Zhi. 2020;56(10):726-9.

16. Jacobi C. Dietrich T, Cursiefen C, et al Das trockene Auge. Aktuelle Konzepte zu Klassifizierung, Diagnostik und Pathogenese [The dry eye. Current concepts on classification, diagnostics, and pathogenesis]. Ophthalmologe. 2006;103(1):9-17.

17. Mclaughlin S. Welch J, Macdonald E, et al Xerophthalmia-a potential epidemic on our doorstep? Eye. 2014; 28(5): 621-3.

18. Management and Therapy of Dry Eye Disease: Report of the Management and Therapy Subcommittee of the International Dry Eye WorkShop (2007). The Ocular Surface. 2007; 5(2): 163178..

19. Ma M. Yuan Q, Ye L, et al An experimental study of amniotic lacrimal duct stents in the treatment of perimenopausal female rabbits with dry eye. Mol Med Rep. 2019;19(2):1056-64.

20. Pepping F. Kavishe F P, Hackenitz EA, et al Prevalence of xerophthalmia in relation to nutrition and general health in preschool-age children in three regions of Tanzania. Acta Paediatr. 2010; 77(6): 895-906.

21. Brian G. Tousignant B, Venn BJ, et al Serum Retinol and Xerophthalmia Among a Prison Population in Papua New Guinea. Ophthalmic Epidemiol. 2011; 18(6): 288-94.

22. Lu F. Tao A, Hu Y. Tao W, Lu P. Evaluation of Reliability and Validity of Three Common Dry Eye Questionnaires in Chinese. J Ophthalmol. 2018 27; 2018:2401213..

23. Chien KJ. Horng CT, Huang YS. Hsieh YH, Wang CJ. Yang JS, Lu CC. Chen FA. Effects of Lycium barbarum (goji berry) on dry eye disease in rats. Mol Med Rep. 2018;17(1):809-18.

24. Gassmann B. Control of Vitamin A Deficiency and Xerophthalmia. Report of a Joint WHO/UNICEF/USAID/Helen Keller International/IVA CG Meeting. 74 Seiten, 7 Abb. 15 Tab. WHO Technical Report Series 672. World Health Organization, Geneva 1982. Preis: 7,- sfrs. Molecular Nutrition \& Food Research. 2010; 26(9): 837-837..

25. Jiang Y. Yang C, Zheng Y. Liu Y, Chen Y. A Set of Global Metabolomic Biomarker Candidates to Predict the Risk of Dry Eye Disease. Front Cell Dev Biol. 2020;8:344. 
26. Geerling G. Tost FHW. Surgical occlusion of the lacrimal drainage system. Dev Ophthalmol. 2008; 41: 213-29.

27. Yang J. Li M, Xiao XL. Efficacy of artificial tears for children xerophthalmia. International Eye ence. 2016; 16(3): 569-71.

28. Jones $C E$. Anklesaria $M$, Gordon $A D$, et al Retrospective safety study of the herrick lacrimal plug: a device used to occlude the lacrimal canaliculus. CLAO J. 2002;28(4):206 - 10.

\section{Figures}

\section{Figure 1}

Model 6303 preloaded punctal plug produced by OASIS Medical, USA. 


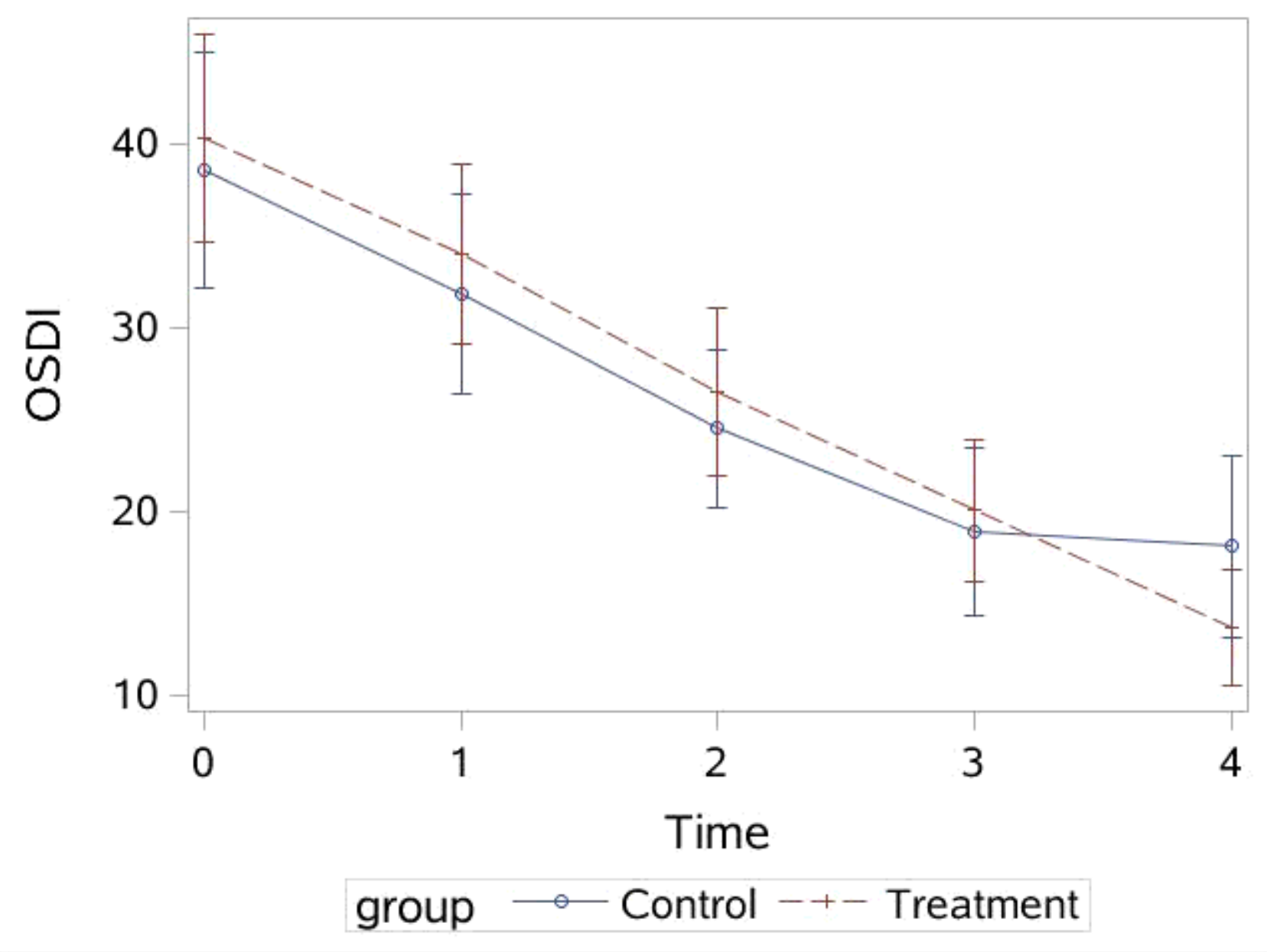

Figure 2

Comparison of OSDI scores between the two groups. x-axis: Before the lacrimal canalicular embolization and 1 week, 1 month, 3 months, and 6 months after the embolization. There was no statistically significant difference in OSDI scores between the experimental group and the control group at 6 months after treatment $(p=0.53)$. 


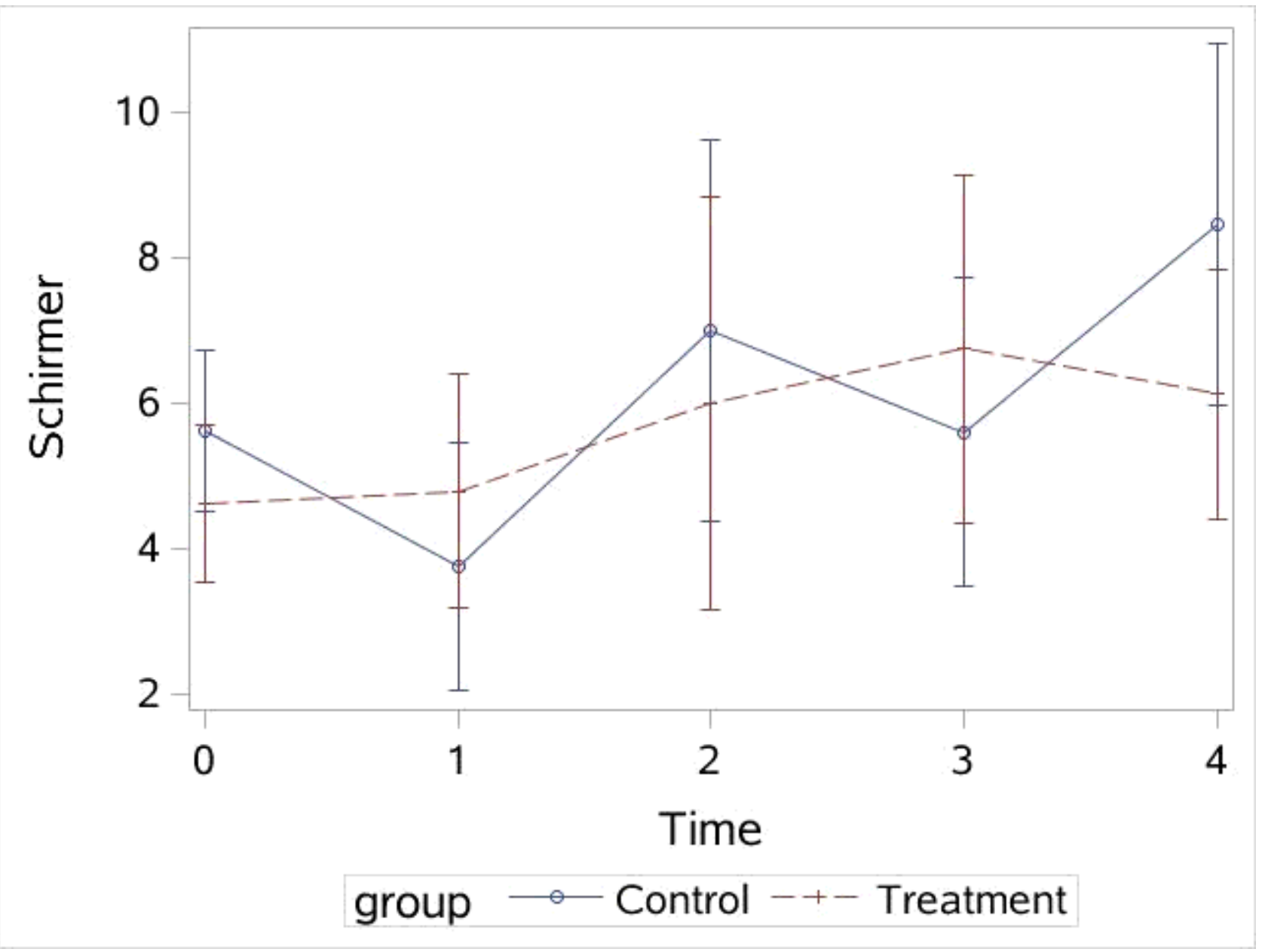

Figure 3

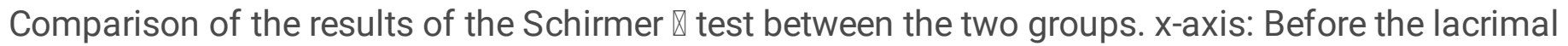
canalicular embolization and 1 week, 1 month, 3 months, and 6 months after the embolization. There was no statistical difference between the experimental group and the control group in the Schirmer $\otimes$ test 6 months after treatment $(p=0.41)$. 


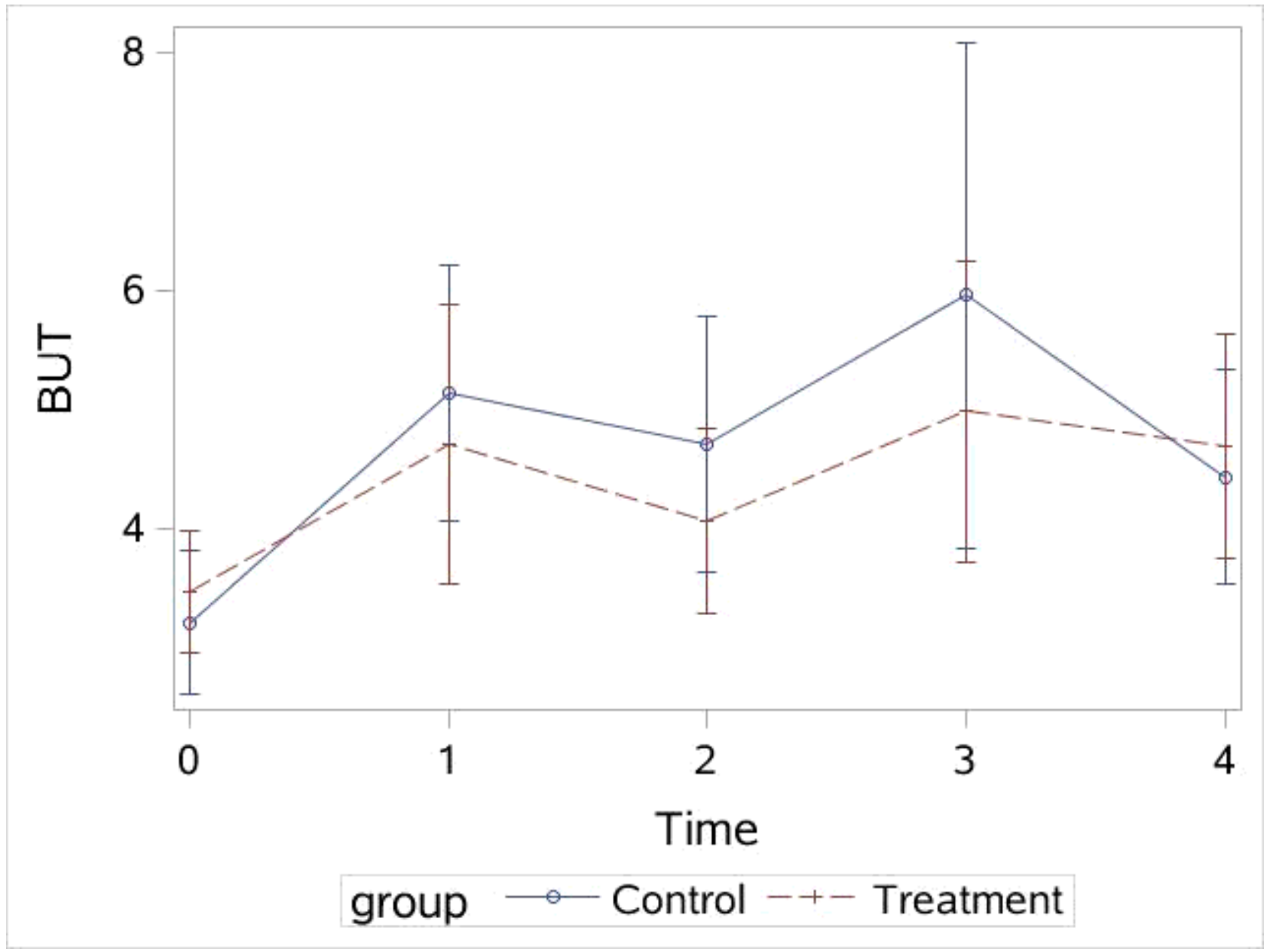

Figure 4

Comparison of BUT results between the two group. x-axis: Before the lacrimal canalicular embolization and 1 week, 1 month, 3 months, and 6 months after the embolization. There was no statistically significant difference in BUT levels between the experimental group and the control group 6 months after treatment $(p=0.35)$. 